\title{
Energetics and electronic structure of tubular Si vacancies filled by carbon nanotubes
}

\author{
Taketo Kochi ${ }^{1 *}$ and Susumu Okada ${ }^{1 \dagger}$ \\ ${ }^{1}$ Graduate School of Pure and Applied Sciences, University of Tsukuba, Tsukuba, Ibaraki 305-8571, Japan
}

\begin{abstract}
We studied the energetics and electronic structure of Si nanoscale vacancies incorporating a carbon nanotube (CNT), using first-principles total-energy calculations based on the density functional theory. Our calculations show that the incorporated CNT in the Si nano-tunnel acts as an atom-thickness liner providing the electrostatically flat nanoscale space inside the CNT by shielding the dangling bond states of tubular $\mathrm{Si}$ vacancies. The incorporation of CNT into the tubular $\mathrm{Si}$ vacancies is exothermic with the energy gain up to $7.4 \mathrm{eV} / \mathrm{nm}$ depending on the diameter of the vacancy and encapsulated CNT. The electronic states of the vacancy substantially hybridize with those of the CNT, leading to complex electronic energy band near the Fermi level.
\end{abstract}

\section{Introduction}

Nanoscale materials are known to exhibit wide variation in electronic structure depending on their dimensionality and topology. By imposing the appropriate one-dimensional boundary conditions on graphene, tubular forms of honeycomb networks of carbon atoms are known to be either a metal or a semiconductor depending on the atomic arrangement along their circumference. ${ }^{1-3)}$ In the case of networks comprising hexagons and 12 pentagons with zero-dimensional boundary conditions, we can construct hollowcage $\mathrm{C}$ clusters, namely fullerenes, which possess bunched energy levels in their $\pi$ electron states, characterized by spherical harmonics $Y_{l m}$, because of the approximately spherical distribution of electrons throughout the networks. ${ }^{4-8)}$ Furthermore, these nanoscale materials can be constituent units for the various condensed phases possessing interesting morphologies and fascinating physical properties by assembling them with appropriate synthesis condition as the case of atoms in conventional solids. In some cases, the electronic structure of condensed phases is not the simple sum of those of each constituent due to the substantial interaction between them. ${ }^{9-13)}$

\footnotetext{
${ }^{*}$ E-mail address: tkochi@comas.frsc.tsukuba.ac.jp

${ }^{\dagger}$ E-mail address: sokada@comas.frsc.tsukuba.ac.jp
} 
From an opposite view, nanoscale vacancies in semiconducting materials can be regarded as a negative version of nanoscale clusters or molecules, which also exhibits various electronic properties depending on their sizes and shapes as the nanoscale positive materials. ${ }^{14-18)}$

Due to the low dimensionality and nanoscale size of vacancies, the vacancies in semiconductors are applicable for electronic, optical, and optoelectronic devices. ${ }^{19,20)}$ Because of the structural similarity between the nanoscale vacancies and nanoscale materials, the nanoscale vacancies can form various hybrids consisting of negative and positive versions of nanoscale constituents by accommodating nanoscale materials into the nanoscale vacancies. The resultant hybrid structure may exhibit interesting physical properties, depending on the combination of the negative and positive nanoscale constituents as in the case of condensed phases of nanoscale materials. Indeed, vacancies in GaAs filled by $\mathrm{C}_{60}$ have been synthesized by the molecular beam epitaxy experiment acting as quantum dots. ${ }^{21)}$ Moreover, a recent experiment has reported the single-electron tunneling through $\mathrm{C}_{60}$ in a metal-insulator-semiconductor structure. ${ }^{22}$ ) However, it is still uncertain whether the electronic properties of the hybrids of vacancies and nanomaterials is the simple sum of those of negative and positive constituent units or not.

In the present work, we aim to give a theoretical insight into fundamental properties of the hybrid systems consisting of nanoscale vacancies and materials. Here, we focus on a tubular Si vacancy filled by a CNT, as a representative hybrid system consisting of negative and positive nanostructures. Our calculations showed that the incorporation of CNT into the tubular vacancy with appropriate spacing is exothermic with energy gain of a few eV/nm. The CNT incorporated in the Si nano-tunnel acts as the liner which level the undulation of the electrostatic potential in the nano-tunnel arising from the unsaturated covalent bonds of the Si vacancy at the surface of the tunnel. According to the substantial hybridization between the dangling bond states of Si and $\pi$ states of CNT, the electronic structure of the hybrid system is not the simple sum of those of the vacancy and CNT depending on the combination of the diameter of the vacancy and CNT. The fact indicates that the hybrid system may provide the electronically smooth nanospace in conventional semiconductors. 


\section{Method and models}

All calculations were performed based the density functional theory ${ }^{23,24)}$ using a STATE package. ${ }^{25}$ We used local density approximation (LDA) to express the exchangecorrelation energy of interacting electrons. ${ }^{26,27)}$ Ultrasoft pseudopotentials were adopted for describing the interaction between nuclei and valence electrons. ${ }^{28)}$ The valence wave functions and charge density were expanded with a plane-wave basis set with cutoff energies of 25 and $225 \mathrm{Ry}$, respectively. We excavated nano-tunnels with diameters of $10,11,12$, and $13 \AA$ thorough a Si slab with $23.5 \AA$ thickness of which surfaces are fully terminated by $\mathrm{H}$ atoms. To impose the commensurability condition between $\mathrm{Si}$ and CNT, the tunnel is parallel to [130] direction which corresponds to the double periodicity of zigzag CNTs. For the incorporated CNT into the tunnel, we consider $(8,0)$, $(9,0),(10,0)$, and $(11,0)$ CNTs. According to the choice of the tunnel direction and CNT species, lateral cell parameters of the slab are $a=25.76$ and $b=8.58 \AA$ containing up to about $300 \mathrm{Si}$ and $90 \mathrm{C}$ atoms. Under the lateral cell parameters, a unit cell along the tube axis was slightly elongated in this direction by $0.1 \%$ which does not affect the energetics of an isolated CNTs. An equidistance $1 \times 4 \times 1-\boldsymbol{k}$ mesh was taken for the Brillouin zone integration.

\section{Results and discussion}

Figure 1 shows optimized tures of the Si slabs with tubular vacancies and tubular vacancies with CNT lining. For both tunnels without and with CNT lining, substantial reconstruction of Si atoms situated at the surface of tunnels occurs to reduce the number of dangling bond states. In spite of the structural reconstruction of Si atoms, the surfaces of the Si tunnel still have unsaturated covalent bonds. In contrast to the substantial structural reconstruction of Si atoms, CNTs almost retain their circular shape except the cases in which the inner space of the tunnel is insufficient to accommodate CNTs. For the tunnel with the diameter of $10 \AA$, due to the small inner space, encapsulated CNTs are deformed from the circular cross section due to the formation of the covalent bonds with the Si atoms at the tunnel surfaces. Interestingly, although the spacing between walls of CNT and of tunnel is large, the $(8,0) \mathrm{CNT}$ is substantially deformed compared with thicker CNTs.

To give further insight into the energetics of the tubular Si vacancies without and with the CNT incorporated, we evaluate the formation energies of Si vacancies and incorporation energy of CNT in the vacancies. Figure 2(a) shows the formation energy 
$\mathrm{Si}$

(a)

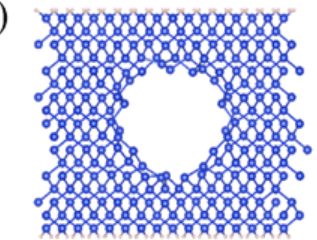

$\mathrm{Si}$

(b)

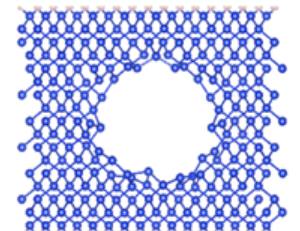

$\mathrm{Si}$

(c)

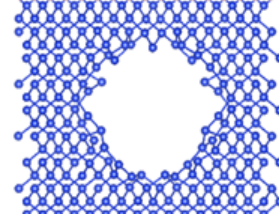

$\mathrm{Si}$

(d)
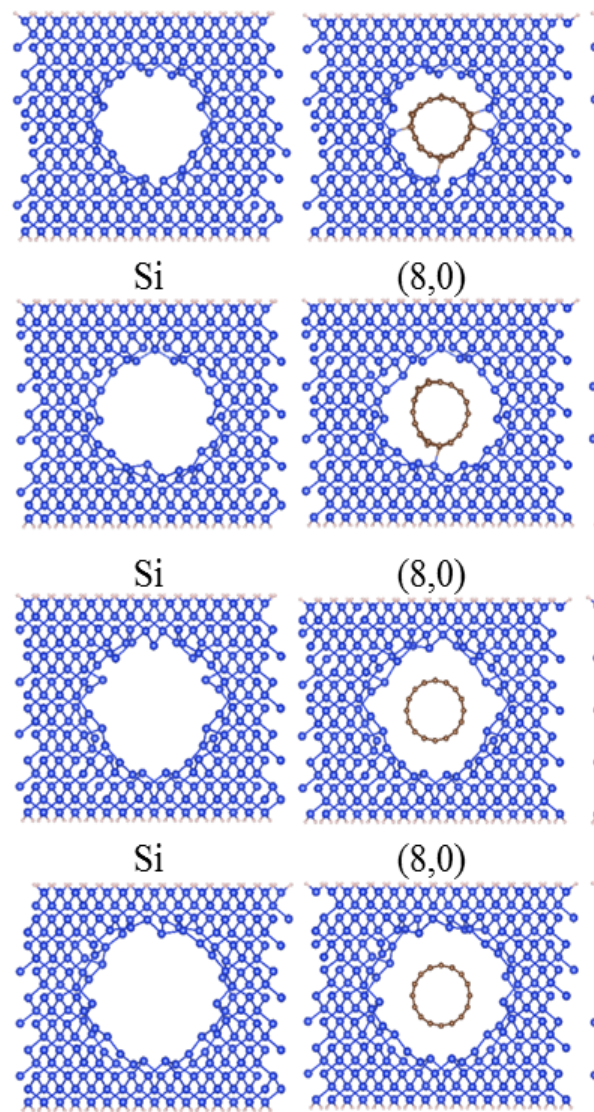

$(8,0)$

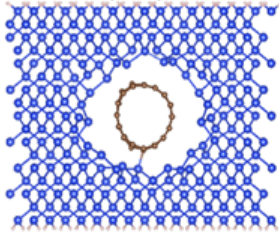

$(8,0)$
$(9,0)$

$(10,0)$

$(11,0)$

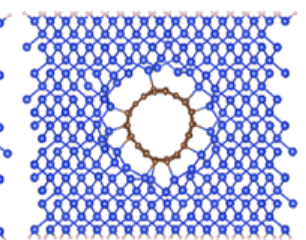

$(9,0)$

$(10,0)$
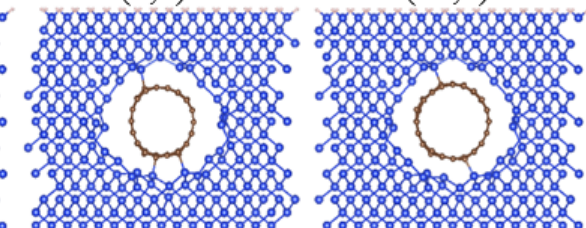

$(11,0)$

$(9,0)$

$(10,0)$
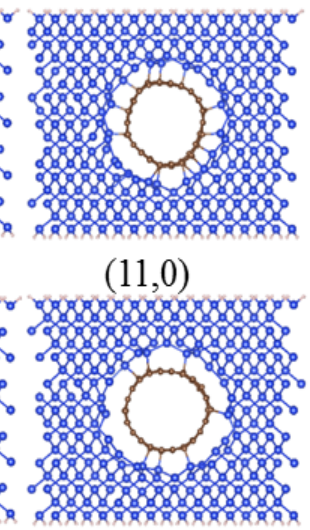

$(11,0)$

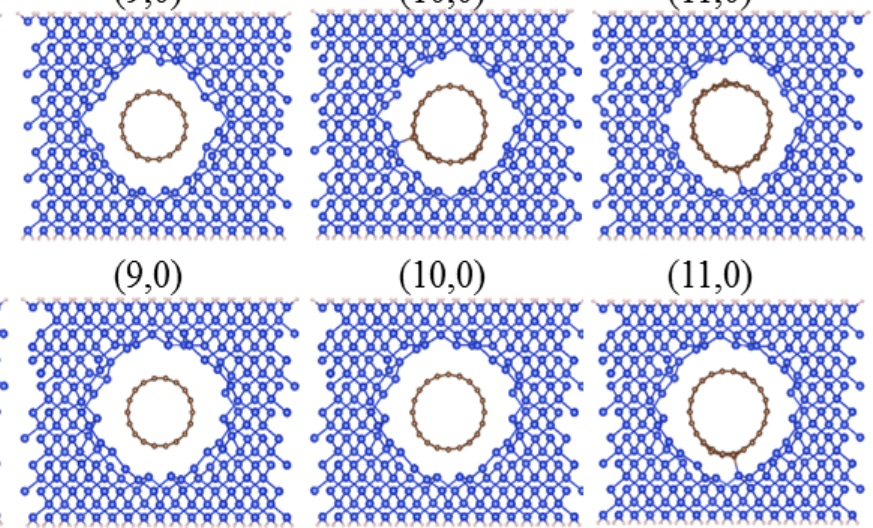

Fig. 1. Optimized structures of tubular Si vacancies with diameters of (a)10, (b)11, (c)12, and (d) $13 \AA$. In each figure, optimized structures of tubular vacancies with $(8,0),(9,0),(10,0)$, and $(11,0)$ CNTs are depicted from left to right. Blue, brown, and white circles denote $\mathrm{Si}, \mathrm{C}$, and $\mathrm{H}$ atoms, respectively.

of Si vacancies as a function of the vacancy diameter. The formation energy is defined as

$$
E=E_{\mathrm{V}}+n \mu_{\mathrm{Si}}-E_{\mathrm{Si}}
$$

where $E_{\mathrm{V}}, E_{\mathrm{Si}}, \mu_{\mathrm{Si}}$, and $n$ denote the total energy of $\mathrm{Si}$ with tubular vacancies, the total energy of the pristine Si slab, the chemical potential of Si atom in their bulk form, and the number of Si atoms removed from the slab, respectively. The formation energy monotonically increases with increasing the diameter of the vacancy studied here. On the other hand, the second energy difference with respect to the diameter seems to possess peaks at particular diameters, because of the convex curve of the formation energy. These results imply that the tubular vacancies possess the magic number of 
their diameter as the case of the zero-dimensional vacancies arising from the number of dangling bonds at the surfaces. ${ }^{29,30)}$

Figure 2(b) shows the incorporation energy of CNT in tubular Si vacancies as a function of the vacancy diameter. The incorporation energy of CNT in the tubular Si vacancy is defined as

$$
\Delta E=E_{\mathrm{CNT} / \mathrm{V}}-E_{\mathrm{V}}-E_{\mathrm{CNT}}
$$

where $E_{\mathrm{CNT} / \mathrm{V}}, E_{\mathrm{V}}$, and, $E_{\mathrm{CNT}}$ are total energies of the vacancies with CNT, vacancies, and CNT, respectively. For the most of CNT, the incorporation reaction is exothermic with the energy gain of a few eV per nm depending on the diameters of vacancies and CNTs except the $(11,0) \mathrm{CNT}$ in the vacancy with the diameter of $10 \AA$. In the case of $(11,0) \mathrm{CNT}$ for the vacancy with the diameter of $10 \AA$, the inner space of the vacancy is insufficient to accommodate $(11,0) \mathrm{CNT}$ due to its large diameter $(8.4 \AA)$. For $(8,0)$ and $(9,0) \mathrm{CNTs}$, the incorporation energy monotonically decreases with increasing the diameter of the vacancies from 10 to $13 \AA$. The fact indicates that the CNTs can be accommodated in narrower vacancies. For $(10,0) \mathrm{CNT}$, the energy possesses a minimum value of the diameter of $12 \AA$. These results imply that the incorporation energy depends on the distance between $\mathrm{C}$ and $\mathrm{Si}$ atoms. In addition, it should be noted that the large incorporation energy for the thin CNTs indicates the substantial interaction between Si and these CNT.

Figure 3 shows contour plots of the electrostatic potential of the clean tubular Si vacancies and vacancies filled by the CNT. In the case of the clean vacancies, inhomogeneous potential profile is observed, owing to the Si dangling bonds at the surface of vacancies. This potential undulation may cause the modulation of physical properties of the molecules inserted into the nanoscale tubular spacing in Si. On the other hand, interestingly, the almost flat potential profile emerges inner space of CNTs, although the dangling bond state retain upon CNT incorporation. Thus, the incorporated CNT into Si vacancies levels the electrostatic potential of the nanoscale tubular space by shielding the effects of the dangling bond states of the Si vacancies. This trend does not depend on the distance between $\mathrm{C}$ and Si atoms. Thus, the molecules or atoms inserted into the inner space of CNT incorporated into Si vacancies are unlikely affected by the dangling bond of Si vacancies. Note that the potential undulation still emerges in the void between CNT and Si surfaces, because of the number of the unsaturated covalent bonds. 



Fig. 2. (a) Formation energies of tubular and (b) incorporation energies of CNT in the tubular vacancies as a function of the vacancy diameters. In (b), triangles, rhombuses, circles, and squares denote the incorporation energy for $(8,0),(9,0),(10,0)$ and $(11,0) \mathrm{CNT}$, respectively.

Figure 4 shows the counter plots of the charge density of the Si vacancies and those incorporating CNTs. For the case of the vacancies whose diameter is relatively small for the CNT incorporation, the finite charge density emerges between $\mathrm{C}$ and Si atoms, indicating the formation of covalent bond between CNT and vacancy surfaces, which might modulate electrostatic potential inside the vacancies and the electronic structures of CNT incorporated in the vacancies. A previous theoretical calculation has shown that CNT adsorbed on the Si surfaces forms covalent bonds with the step edge Si atoms. ${ }^{31)}$ In contrast, for the vacancies with large diameter, filled by CNTs with small diameter, charge density is absent at the space between CNT and the surfaces, but CNT retains their cylindrical charge distribution, indicating that the incorporation does not affect the electron system of CNTs.

Figure 5 shows the electronic energy bands of the tubular Si vacancies and those incorporating CNTs. The electrostatic potential modulation upon the CNT incorporation and the bond formation between CNT and vacancies imply the substantial modulation of the electronic structures of vacancies incorporating CNTs. Indeed, for the most of cases, electronic structure of CNT inserted in the Si vacancy does not exhibit characteristic dispersive bands of CNTs due to the substantial interaction between CNT 
(a)

$\mathrm{Si}$

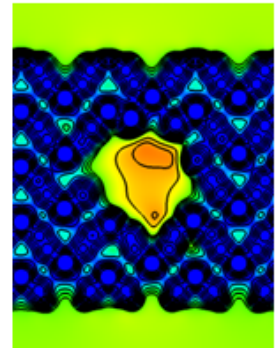

$\mathrm{Si}$

(b)

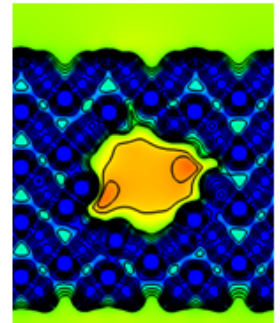

$\mathrm{Si}$

(c)



(d)

$$
\mathrm{Si}
$$

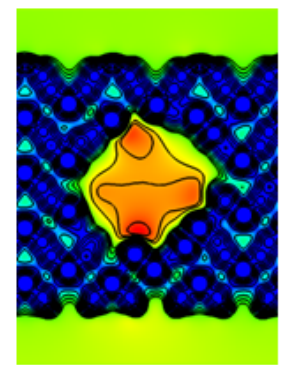

$(8,0)$

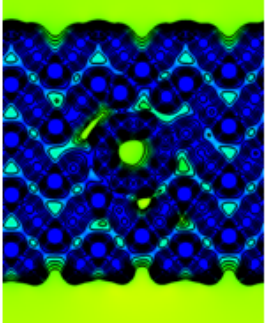

$(8,0)$

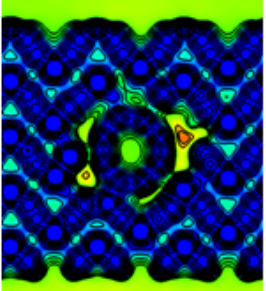

$(8,0)$

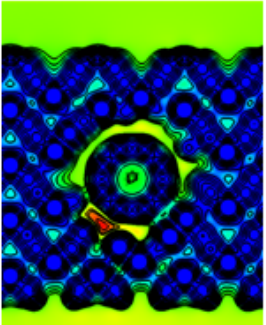

$(8,0)$

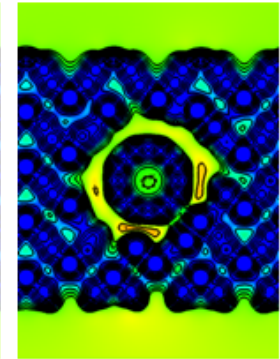

$(9,0)$

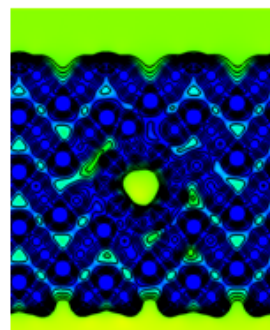

$(9,0)$

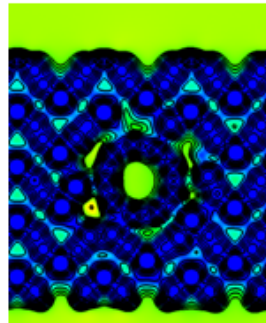

$(9,0)$

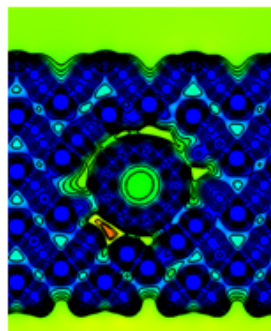

$(9,0)$

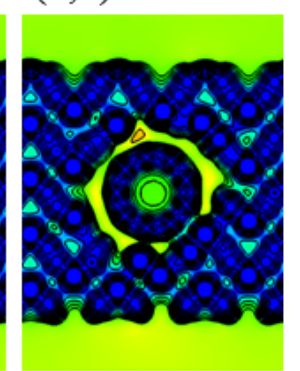

$(10,0)$

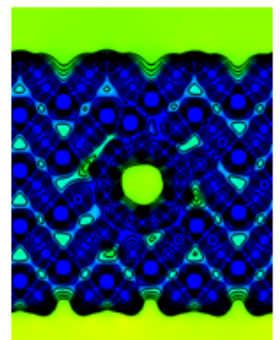

$(10,0)$

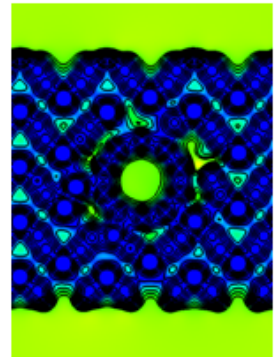

$(10,0)$

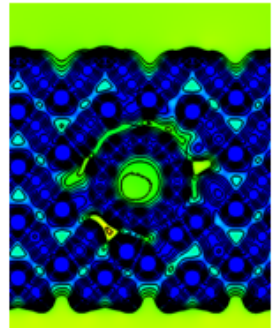

$(10,0)$



$(11,0)$

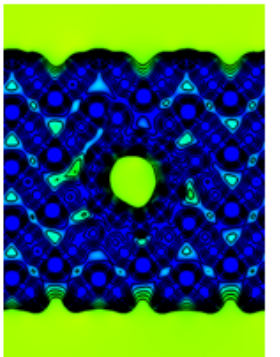

$(11,0)$

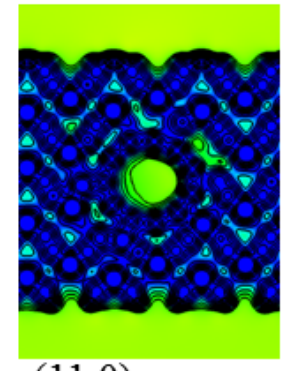

$(11,0)$



$(11,0)$

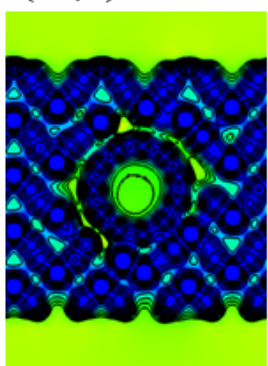

Fig. 3. Counter plots of the electrostatic potential of clean and CNT-filled Si vacancies with diameters of (a)10, (b)11, (c)12, and (d)13. .

and vacancies except the vacancies with diameter of $13 \AA$ incorporating $(8,0)$ and $(9,0)$ CNTs. Wave functions of the CNT inserted into Si vacancies near the Fermi level exhibit hybridized nature between them, making us difficult to assign whether the states belong to CNT or not. The fact indicates that the electron system of the hybrid systems comprising CNT and Si vacancies may exhibit unusual properties those are absent in the simple sum of the electronic properties of the CNT and Si vacancies. Due to the 


\section{$\mathrm{Si}$}

$(8,0)$

$(9,0)$

$(10,0)$

(a)

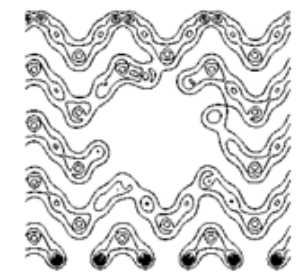

$\mathrm{Si}$



$(8,0)$

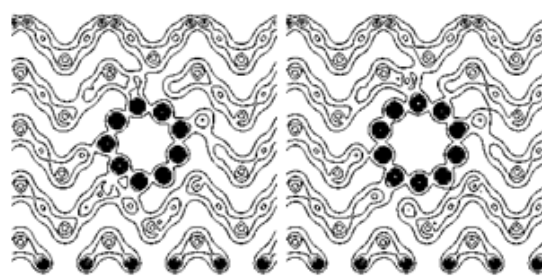

$(9,0)$

$(10,0)$



$(9,0)$

$(10,0)$

$(11,0)$
$(11,0)$

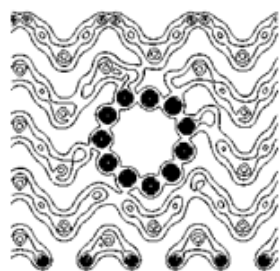

$(11,0)$

(b)
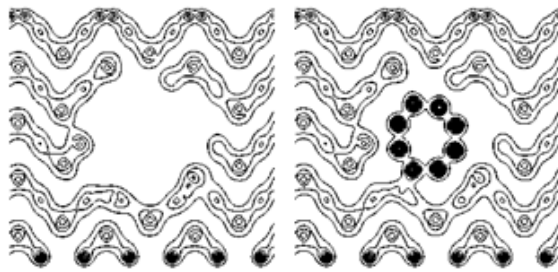

$\mathrm{Si}$

$(8,0)$

(c)
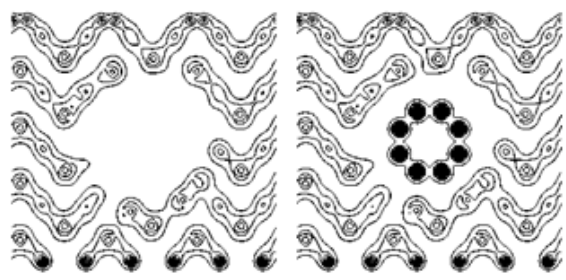

$\mathrm{Si}$
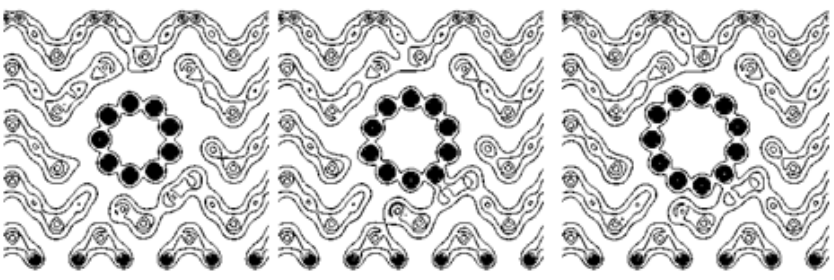

$(9,0)$

$(10,0)$

$(11,0)$

(d)
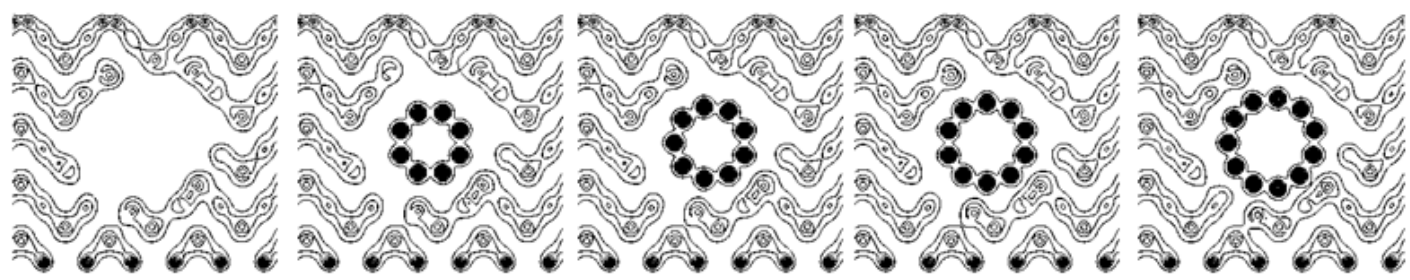

Fig. 4. Counter plots of the charge density of tubular vacancies with the diameters of (a)10, (b)11, (c) 12 , and (d)13 $\AA$, which incorporate $(8,0),(9,0),(10,0)$, and $(11,0)$ CNTs.

hybridized nature, CNTs incorporated in Si vacancies may not show the high carrier mobility as an isolated case, but act as the conventional one-dimensional conducting wire with the number of carriers. On the other hand, perhaps, CNT is more likely appropriate for the liner of Si nano-tunnels, providing the electrostatically homogeneous nano-space in their inner space, as stated above.

\section{Summary}

We studied the energetics and electronic structure of the one-dimensional nanoscale tunnel through $\mathrm{Si}$, which is filled by a CNT using the density functional theory with 


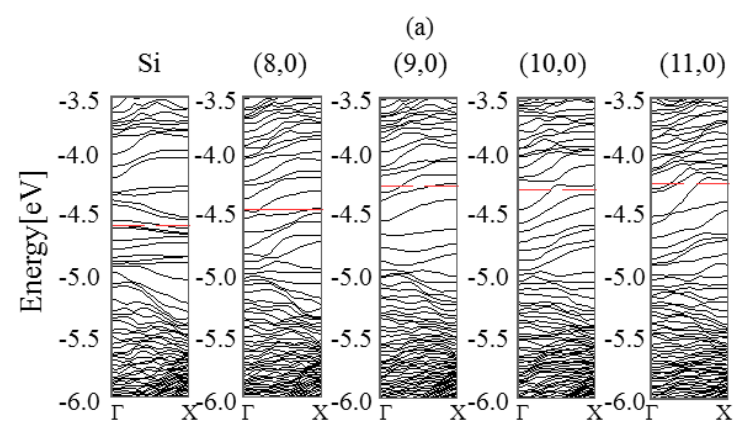

(c)

$\mathrm{Si} \quad(8,0)$

$(9,0)$

$(10,0)$

$(11,0)$

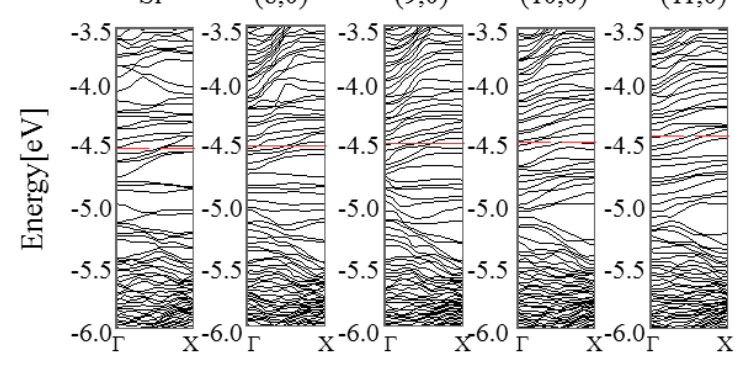

(b)

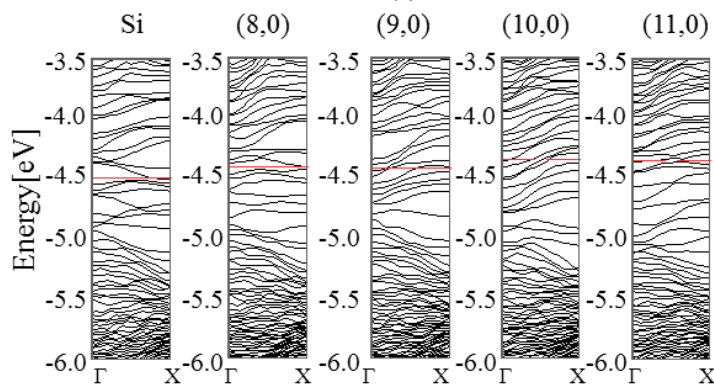

(d)

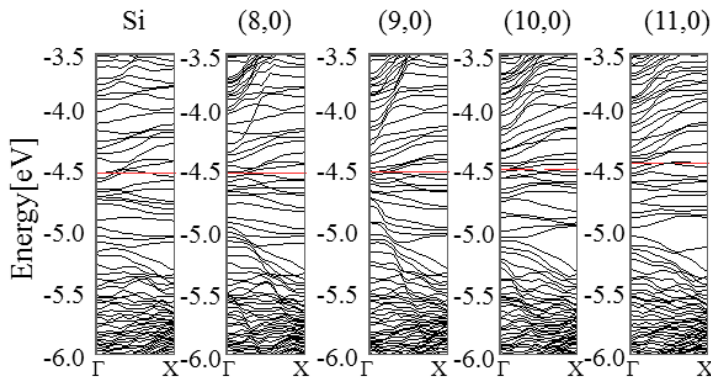

Fig. 5. Electronic energy bands of tubular vacancies with diameters of (a)10, (b)11, (c)12, and (d)13 A, which contain $(8,0),(9,0),(10,0)$, and $(11,0)$ CNTs. The Fermi Level is denoted by a horizontal dotted line.

the local density approximation. Our calculations showed that the formation energy of the tubular Si vacancy with CNT lining strongly depends on the diameter of the vacancy and CNT. For all CNTs in vacancies except a $(11,0) \mathrm{CNT}$ in a vacancy with the diameter of $10 \AA$, the incorporation reaction of the CNT in the vacancy is exothermic indicating that the hybrid structure is energetically stable: The CNTs with small diameter results in the large incorporation energy compared with the CNT with larger diameter in the vacancies with appropriate diameter which results in about $3 \AA$-spacing between the CNT wall and vacancy. The electronic structure of CNT is highly modulated by the hybridization with the electronic states associated with the unsaturated covalent bonds at the surface of vacancy. The fact indicates that the nanoscale tunnel with CNT lining is the novel one-dimensional electron system which is characterized by the complex hybridization between $\pi$ states of the CNT and dangling bond states of $\mathrm{Si}$ vacancies. Furthermore, the incorporated CNT into the Si nano-tunnel acts as the liner which level the undulation of the electrostatic potential in the nano-tunnel arising from the unsaturated covalent bonds of Si situated at the surface of the tunnel by the hybridization. Thus, the hybrid system may provide the electrostatically smooth nanospace in conventional semiconductors. 


\section{Acknowledgements}

This work was supported in part by a Grant-in-Aid for Scientific Research from the Ministry of Education, Culture, Sports, Science and Technology of Japan and the Joint Research Program on Zero-Emission Energy Research, Institute of Advanced Energy, Kyoto University. Computations were performed on a NEC SX-8/4B at the University of Tsukuba, a SGI ICE XA/UV at the Institute for Solid State Physics, The University of Tokyo, and a NEC SX-Ace at the Cybermedia Center, Osaka University. 


\section{References}

1) S. Iijima, Nature 354, 56 (1991).

2) N.Hamada, S.Sawada, and A.Oshiyama, Phys. Rev. Lett. 68, 1579 (1992).

3) R. Saito, M. Fujita, G. Dresselhaus and M. S Dresselhaus, Appl. Phys. Lett. 60, 2204 (1992).

4) H.Kroto, J.R. Heath, S.C. O'Brien, R.F. Curl, and R.E. Smalley, Nature 318162 (1985).

5) W. Krätschmer, K. Fostiropoulous, and D. R. Hoffman, Chem. Phys. Lett. 170, 167 (1990).

6) W. Krätschmer, L. D. Lamb, K. Fostiropoulous, and D. R. Hoffman, Nature 347, 354 (1990).

7) S. Saito, A. Oshiyama, Phys. Rev. Lett. 66, 2637 (1991).

8) S. Saito, S. Okada, S. Sawada, and N.-I. Hamada, Phys. Rev. Lett. 75, 685 (1995).

9) B. W. Smith, M. Monthioux, and D. E. Luzzi, Nature 396, 323 (1998).

10) B. Burteaux, A. Claye, B. W. Smith, M. Monthioux, D. E. Luzzi, and J. E. Fischer, Chem. Phys. Lett. 310, 21 (1999).

11) K. Hirahara, K. Suenaga, S. Bandow, H. Kato, T. Okazaki, H. Shinohara, and S. Iijima, Phys. Rev. Lett. 85, 5384 (2000).

12) S. Okada, S. Saito, and A. Oshiyama, Phys. Rev. Lett. 86, 3835 (2001).

13) M. Otani, S. Okada, and A. Oshiyama, Phys. Rev. B 68, 125424 (2003).

14) T. Akiyama, A. Oshiyama, and O. Sugino, J. Phys. Soc. Jpn. 67, 4110 (1998).

15) T. Akiyama, Y. Okamoto, M. Saito, and A. Oshiyama, Jpn. J. Appl. Phys. 38, L1363 (1999).

16) T. Akiyama and A. Oshiyama, J. Phys. Soc. Jpn. 70, 1627 (2001).

17) O. Sugino and A. Oshiyama, Phys. Rev. Lett. 23, 1858 (1992).

18) O. Sugino and A. Oshiyama, Phys. Rev. B 42, 11869 (1990).

19) J. Neugebauera and CG Van de Walle, Appl. Phys. Lett. 69, 503 (1996).

20) J.Wolters, A W. Schell, G. Kewes, N. Nüsse, M. Schoengen, H. Döscher, T. Hannappel, B. Löchel, M.Barth and O. Benson, Appl. Phys. Lett. 97, 141108 (2010).

21) J. Nishinaga and Y. Horikoshi, J. Cryst. Growth 323, 135 (2011).

22) R. Hayakawa, N. Hiroshiba, T. Chikyow, and Y. Wakayama, Adv. Funct. Mater. 21, 2933 (2011). 
23) P. Hohenberg and W. Kohn, Phys. Rev. 136, B864 (1964).

24) W. Kohn and L. J. Sham, Phys. Rev. 140, A1133 (1965).

25) Y. Morikawa, K. Iwata, and K. Terakura, Appl. Surf. Sci. 169, 170, 11 (2001).

26) J. P. Perdew and A. Zunger, Phys. Rev. B 23, 5048 (1981).

27) D. M. Ceperley and B. J. Alder, Phys. Rev. Lett. 45, 566 (1980).

28) D. Vanderbilt, Phys. Rev. B 41, 7892 (1990).

$29)$ D. J. Chadi and K. J. Chang, Phys. Rev. B. 38, 1523 (1988).

30) A. Oshiyama, Mineo Saito and Osamu Sugino, Appl. Surf. Sci. 85, 239 (1995).

31) S. Berber and A. Oshiyama, Phys. Rev. Lett. 96, 105505 (2006). 